\title{
Structure and Dynamics of Nonionic Surfactants Adsorbed at Vacuum/Ionic Liquid Interfaces
}

\author{
M. Dolores Elola* ${ }^{\dagger \dagger}$ and Javier Rodriguez $z^{\ddagger \dagger}$ \\ ${ }^{\dagger}$ Departamento de Física, Comisión Nacional de Energía Atómica, Avenida Libertador 8250, 1429 Buenos Aires, Argentina, and \\ ${ }^{\ddagger}$ ECyT, UNSAM, Martín de Irigoyen 3100, 1650 San Martín, Provincia de Buenos Aires, Argentina
}

\section{Supporting Information}

ABSTRACT: Structural and dynamical properties related to the adsorption of nonionic surfactants at vacuum/ionic liquid interfaces were studied using molecular dynamics simulations. Specifically, the surface activity of pentaethylene glycol monododecyl ether $\left(\mathrm{C}_{12} \mathrm{E}_{5}\right)$ was investigated at the free interface of an imidazolium-based room temperature ionic liquid (RTIL), at different surface densities. At low surface coverages, the incorporation of $\mathrm{C}_{12} \mathrm{E}_{5}$ does not produce meaningful changes in the vacuum/RTIL interface: the $C_{12} E_{5}$ hydrophobic tails remain entangled with those of the RTIL cation groups in the outer shell, whereas the $\mathrm{C}_{12} \mathrm{E}_{5}$ hydrophilic heads reside at an inner layer. At high surface coverages, the structure in the substratein terms of the features exhibited by the local density profiles-
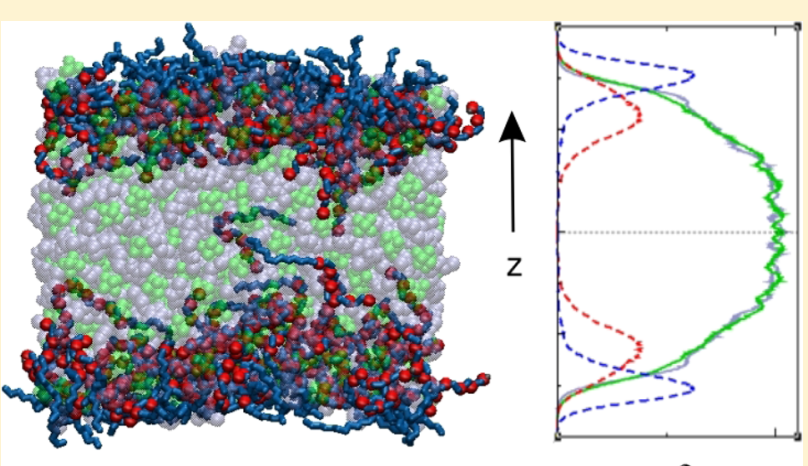

$\rho$ practically vanishes; the interface becomes wider and the surfactant molecules shift toward more external positions. Information about the local structure of the interface at high surface densities can be recovered by performing a tessellation procedure. For the sake of comparison, the surface behavior of two commonly used ionic surfactants, sodium dodecyl sulfate and dodecyl trimethyl ammonium chloride, were also studied. The modifications in the width and structure of the bare vacuum/RTIL interface due to the presence of the ionic surfactants are markedly milder than those observed for the nonionic surfactant. Moreover, the RTIL seemed to behave as a better solvent for the chloride counterions than for sodium ones; which were found to remain bound to the surfactant head groups. An analysis of the dynamics at the surface was also performed. Our results indicate that the presence of increasing amounts of nonionic surfactants leads to a gradual reduction of the mobility of the RTIL species. When ionic surfactants are adsorbed, these retardations are even more severe for the surfactant head groups, where the corresponding diffusion coefficients show reductions of practically 1 order of magnitude.

\section{INTRODUCTION}

In recent years, the subject of chemical reactivity in room temperature ionic liquids (RTILs) has attracted a great deal of attention. ${ }^{1-3}$ These molten salts exhibit a variety of attractive physicochemical properties which make them suitable solvents with practical applications in synthetic processes, ${ }^{4-6}$ photochemistry, ${ }^{7,8}$ chromatography, ${ }^{1,3,9,10}$ separation techniques, ${ }^{11,12}$ catalysis, ${ }^{13,14}$ and electrochemistry, ${ }^{15}$ to cite a few relevant examples.

The consideration of RTIL/vacuum interfaces opens new perspectives for host-environments, with characteristics well differentiated from what is normally perceived in isotropic, bulk phases. At a molecular level, these modifications are the results of the unbalance between the intramolecular forces that prevail at the close vicinity of the interfaces which, in turn, get translated into modifications in local densities, intramolecular connectivities, orientational correlations, and changes in the dynamical properties as well. Such manifestations have been predicted in a series of theoretical studies ${ }^{16-22}$ and corroborated by direct experiments ${ }^{23-29}$ as well. Within this context, the analysis of interactions between amphyphilic molecules and RTILs appears as a natural extension of this previous body of research. Several studies have been conducted in recent years to analyze both the structure and the dynamics of adsorbed nonionic monolayers at free RTILs interfaces. ${ }^{30-32}$ In addition, micellar aggregation processes in the bulk phase of RTILs have been also investigated. ${ }^{32-35}$ To the best of our knowledge, the microscopic description of these previous results has relied exclusively on the direct interpretation of the experimental signals. This motivated us to undertake massive molecular dynamics experiments to gain a complementary insight on the characteristics of the adsorption of a nonionic amphyphile pentaethylene glycol monododecyl ether at the free interfaces of a RTIL sample. In addition, whenever possible, we tried to establish similarities and differences between the present case and the ones involving aqueous substrates or different ionic

\footnotetext{
Received: March 15, 2013

Revised: September 19, 2013

Published: October 4, 2013
} 
surfactants. As we will show, our simulations show a rich variety of behaviors in which the structure and dynamics at the interface is the result of a complex interplay between the different surfactant species and the RTIL components.

The organization of the present work is as follows: In section 2 , we describe the model and the simulation procedure. Section 3 contains the experimental results, and the main conclusions of the paper are summarized in section 4 .

\section{MODEL AND COMPUTATIONAL DETAILS}

The molecular dynamics experiments described in this work were performed on systems comprising nonionic surfactants adsorbed at the free interfaces of a RTIL, 1-butyl-3-methyl imidazolium hexafluorophosphate $\left([\mathrm{BMIM}]^{+}\left[\mathrm{PF}_{6}\right]^{-}\right)$, rectangular slab. More specifically, we analyzed the adsorption of the amphyphile pentaethylene glycol monododecyl ether $\left(\mathrm{C}_{12} \mathrm{E}_{5}\right)$, at different surface coverages. The gross features of the molecular structure of this compound can be cast in terms of two adjacent segments, with hydrophobic and hydrophilic characteristics, respectively. The balance between the lengths of these two moieties determine, to a large extent, its resulting surface activity. However, for the sake of comparison, we also examined the surface behavior of two widely used, anionic and cationic detergents: (i) sodium dodecyl sulfate (SDS) and (ii) dodecyl trimethyl ammonium chloride (DTAC), respectively (see schemes for $\mathrm{C}_{12} \mathrm{E}_{5}, \mathrm{DS}^{-}$and DTA in Figure 1).

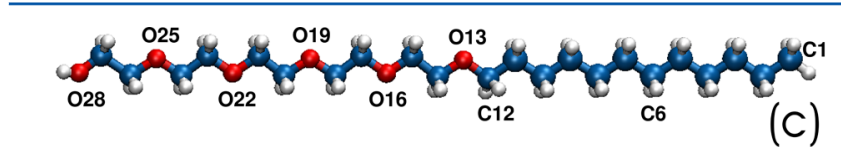

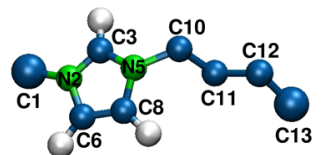

(a)

(b)

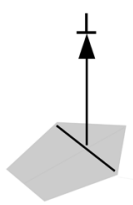

$q\left(\mathrm{SO}_{4}\right)=-1$

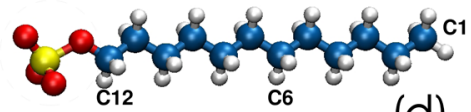

(d)

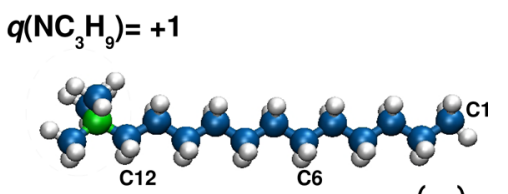

(e)
Figure 1. Schematic representation of (a) 1-butyl 3-methyl imidazolium, $[\mathrm{BMIM}]^{+}$ionic liquid cation; (b) the perpendicular molecular axis in BMIM; (c) nonionic surfactant $\mathrm{C}_{12} \mathrm{E}_{5}$; (d) anionic surfactant $\mathrm{DS}^{-}$; and (e) cationic surfactant $\mathrm{DTA}^{+}$. The total number of sites for each molecule are: 13, 74, 42, and 50 for (a), (c), (d), and (e), respectively.

To facilitate the description that will follow, it will be useful to consider a local system of coordinates, centered at the center of mass of the slab, and oriented so that its two free interfaces are normal to the $z$-axis. The lengths of the simulation box were set to $L_{x}=L_{y}=70 \AA$ and $L_{z}=150 \AA$, whereas the width of the liquid slab was kept close to $50 \AA$ A. Periodic boundary conditions were applied along the three main axes. The starting slab configurations were built from previously equilibrated, $70 \times 70 \times 50 \AA^{3}$ bulk samples of the pure RTIL, containing $N_{\text {ip }}$ ion pairs, in which the length of the simulation box along the $z$-axis was gradually expanded. After reaching equilibrium for $L_{z}=150 \AA, N_{\mathrm{s}}$ surfactant molecules were randomly distributed at each of the two free interfaces, at appropriate surface densities $\rho_{\text {surf }}$ with their hydrophilic headgroups in close contact with the underlying substrate, and with axial molecular orientations mostly parallel to the $z$-axis (see Figure 2).

There are a variety of force fields available in the literature to model interactions involving imidazolium-based ionic liquids. ${ }^{36-38}$ In the present study, we adopted the flexible model developed by Zhong et (a)

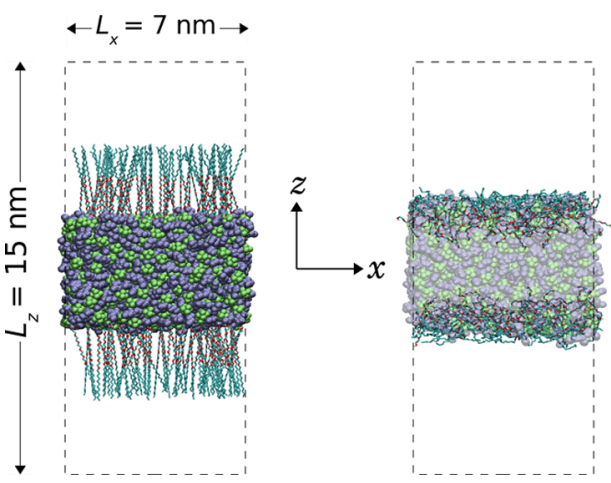

Figure 2. Schematic representation of the system and the simulation box. (a) Initial configuration for an area per surfactant of $78 \AA^{2}$; (b) Equilibrium configuration of the same system after 20 ns. The color code is the following: red and cyan for Oxygen and Carbon sites of $\mathrm{C}_{12} \mathrm{E}_{5}$; blue for $[\mathrm{BMIM}]^{+}$, green for $\left[\mathrm{PF}_{6}\right]^{-}$. The hydrogen atoms of the surfactants are not represented, for clarity purposes.

al., ${ }^{39}$ which yields reasonable predictions for the structural and dynamical characteristics of RTILs, when compared with direct experimental information. The Hamiltonian is based on a united-atom description of the methyl and butyl groups in the cation so that $[\mathrm{BMIM}]^{+}$is mimicked as a collection of 13 interaction sites (see Figure 1a). In addition, the magnitude of the total charge on the cation and the anion are rescaled down to $0.8 e$, to compensate the lack of the explicit incorporation of polarization contributions. Interactions involving nonionic and ionic surfactants were taken from the CHARMM27 force field. ${ }^{40}$ As usual, the overall potential energy of the system was decomposed into site-site pairwise interactions combining dispersion (Lennard-Jones like) and Coulomb contributions. The standard arithmetic and geometrical means were implemented to model cross-interactions. As a reference, we also undertook a few test runs to examine the adsorption of nonionic surfactants on slabs containing $N_{\mathrm{w}}$ water molecules. In the latter experiments, water interactions were modeled using the SPC/E model. In Table 1, we display the complete list of the systems investigated, along with the corresponding sizes and surface concentrations.

\section{Table 1. Details of the Simulations}

$\begin{array}{cclcccc}\text { system } & \text { interface } & \text { surfactant } & \rho_{\text {surf }}^{-1}\left(\AA^{2}\right) & N_{\text {ip }} & N_{\mathrm{w}} & N_{\mathrm{s}} \\ \text { S0 } & \text { vacuum/RTIL } & \text { vacuum } & & 700 & 0 & 0 \\ \text { S1 } & \text { vacuum/RTIL } & \mathrm{C}_{12} \mathrm{E}_{5} & 490 & 700 & 0 & 10 \\ \text { S2 } & \text { vacuum/RTIL } & \mathrm{C}_{12} \mathrm{E}_{5} & 140 & 700 & 0 & 35 \\ \text { S3 } & \text { vacuum/RTIL } & \mathrm{C}_{12} \mathrm{E}_{5} & 78 & 700 & 0 & 65 \\ \text { S4 } & \text { vacuum/RTIL } & \mathrm{C}_{12} \mathrm{E}_{5} & 50 & 700 & 0 & 100 \\ \text { S5* } & \text { vacuum/RTIL } & \text { SDS } & 78 & 715 & 0 & 65 \\ \text { S6* } & \text { vacuum/RTIL } & \text { DTAC } & 78 & 715 & 0 & 65 \\ \text { W3 } & \text { vacuum/water } & \mathrm{C}_{12} \mathrm{E}_{5} & 78 & 0 & 8085 & 65\end{array}$

The smallest values of $\rho_{\text {surf }}^{-1}$ correspond to cmc experimental values. ${ }^{32,41}$

Dynamical trajectories were generated using the NAMD package ${ }^{42}$ and corresponded to microcanonical runs. The particle mesh Ewald method was implemented to handle long-range Coulomb forces. ${ }^{43,44}$ The equations of motion were integrated using a multiple time step of $1 \mathrm{fs}$ for intramolecular modes, 2 fs for nonbonded, short-ranged forces and $4 \mathrm{fs}$ for the remaining Coulomb forces. Preliminary equilibration trajectories at $T \approx 323 \mathrm{~K}$, lasting typically $\sim 3-5 \mathrm{~ns}$, were discarded. From then on, configurations were saved along $40 \mathrm{~ns}$ trajectories for data analysis. We remark that for the systems involving the largest surfactant concentrations, as well as for those containing ionic surfactants (systems S3, S4, S5*, and S6*), the MD trajectories have been extended up to $200 \mathrm{~ns}$. These long trajectories were necessary in 
order to guarantee the convergence in the computation of structural and dynamical properties, such as the local density profiles and the self-diffusion coefficients. We have checked that no meaningful fluctuations (below 1\%) were observed in all relevant equilibrium ensemble averages after the first $100 \mathrm{~ns}$ period. Evaporation of a couple of molecules was detected only during the preliminary equilibration period. Those molecules were discarded. During the whole length of the equilibrium production run, we have checked that all molecules stayed within the slab-plus-surfactant spatial region.

\section{RESULTS}

3.1. Density Profiles. We will start our analysis by investigating the characteristics of the density fields associated

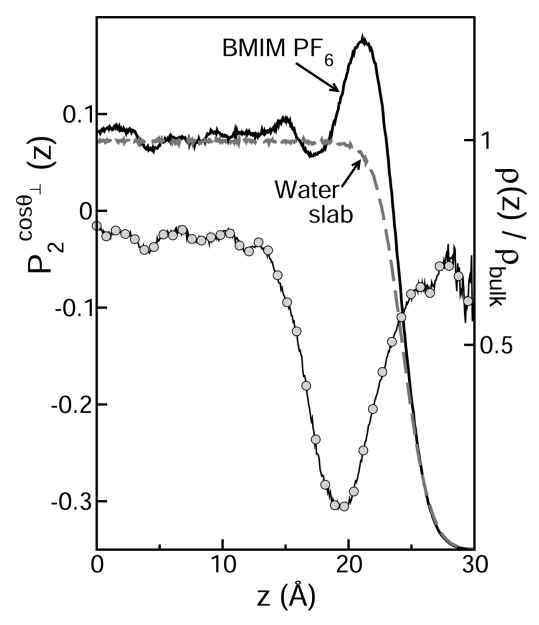

Figure 3. Normalized density profiles along $z$, for the bare vacuum/ RTIL and vacuum/water interfaces (right axis). The second-order Legendre polynomial of the cosine angle between the axis perpendicular to the cation ring and the $z$-axis is displayed with circles (left axis), for the S0 system.

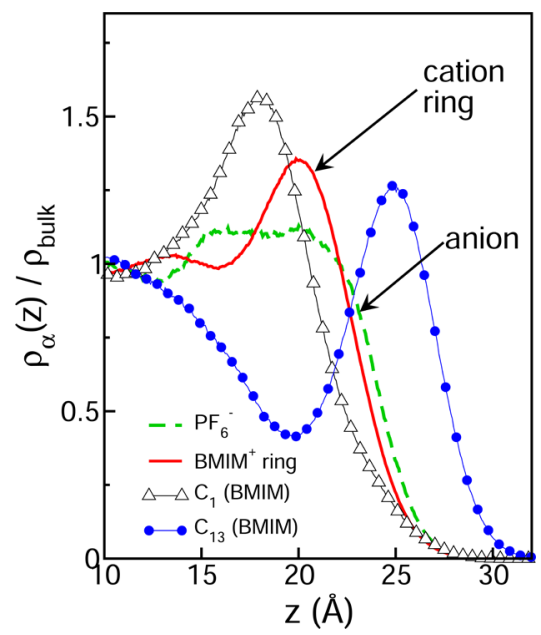

Figure 4. Detail of the density profiles displayed in Figure 3, along the range $10 \leq z \leq 32 \AA$, for different species.

with a set of relevant chemical species. In Figures 3 and 5, we present results for $\rho_{\alpha}(z)$, namely:

$$
\rho_{\alpha}(z)=\frac{1}{A} \sum_{i}\left\langle\delta\left(Z_{i}^{\alpha}-z\right)\right\rangle .
$$
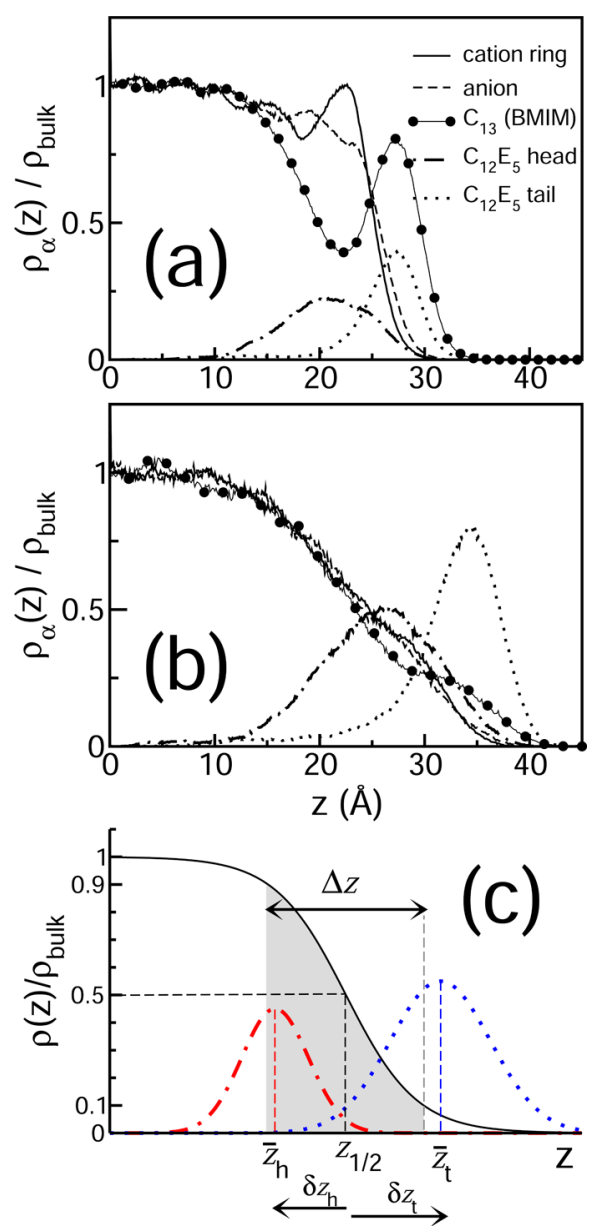

Figure 5. Normalized density profiles of different species along $z$, at different surface coverages of the nonionic surfactant $\mathrm{C}_{12} \mathrm{E}_{5}$. Panels (a) and (b) correspond to the systems S2 and S4, respectively. Solid and dashed lines correspond to cation ring and anion density profiles respectively; dot-dashed and dotted lines represent the surfactant's head and tail densities. The curve corresponding to the terminal methyl, $\mathrm{C}_{13}$, of the butyl group of $[\mathrm{BMIM}]^{+}$has been also included (with circles). The graphic representation of the computed structural parameters is shown in panel (c).

In the previous expression, $\langle\ldots\rangle$ denotes an equilibrium ensemble average and $Z_{i}^{a}$ represents the $z$-coordinate of the $\alpha$-site in the $i$ th molecule. As such, $\rho_{\alpha}(z) \mathrm{d} z$ represents the number of sites of type $\alpha$ per unit area, $A$, along the $x y$-plane, located between $z$ and $z+\mathrm{d} z$. The profiles shown in Figures 3, 4 , and 5 were normalized by the experimental bulk densities at $T=323 \mathrm{~K}$ which, for the case of $[\mathrm{BMIM}]^{+}\left[\mathrm{PF}_{6}\right]^{-}$, corresponds to $\rho_{\text {bulk }}=0.00284 \AA^{-3}\left(1.34 \mathrm{~g} / \mathrm{cm}^{3}\right){ }^{45}$

To gain a more comprehensive picture of the characteristics of the behavior of the different surfactants at vacuum/RTIL interfaces, it will be useful to briefly present an overview of the main characteristics of the bare vacuum/RTIL interface (system S0). We remark that the results that follow agree reasonably well with those reported by Lynden-Bell and Del Pópolo, ${ }^{19}$ using a different force field. The corresponding density profiles are depicted in Figure 3.

The ionic liquid profile (solid line), including contributions from the centers of mass of the cationic and anionic species, looks practically structureless up to $z \approx 12 \AA$. Beyond this distance, the interface exhibits a small depletion at $z \sim 17 \AA$ and a local maximum at $z \sim 22 \AA$, before falling smoothly down to 
zero at $z \approx 27-30 \AA$. Compared to the more common vacuum/water interface (see dashed line in Figure 3) the ionic liquid interface looks clearly much broader. This widening could be somehow anticipated, considering the variety of additional length scales characterizing the intramolecular geometry of the RTIL constituents which, in turn, should modulate the structural characteristics of the interface.

The discrimination between the densities associated to the centers of mass of the anions and two relevant distal sites in the $[\mathrm{BMIM}]^{+}$cations (labeled $\mathrm{C} 1$ and $\mathrm{C} 13$ in Figure 1) provide elements that confirm such hypothesis (see Figure 4). Note that, in doing so, the local structure of the interface is characterized by very prominent, inner and outer peaks (located at $z \approx 18 \AA$ and $z \approx 25 \AA$, associated with $\mathrm{C} 1$ and C13 site densities, respectively), whereas the $\left[\mathrm{PF}_{6}\right]^{-}$profile presents a mild enhancement that prevails in a narrow $15 \AA<z$ $<23 \AA$ interval. In other words, the overall structure can now be cast in terms of an outer hydrophobic region, followed by an inner layer, in which the density of the negatively and positively charged species practically compensate each other.

The analysis of orientational correlations involving the bulkier $[\mathrm{BMIM}]^{+}$groups provide an additional insight. In Figure 3, we present results for local averages of the second order Legendre polynomials of the type:

$$
\overline{P_{2}^{\cos \theta}(z)}=\frac{\sum_{i}\left\langle\delta\left(z-z_{i}\right) P_{2}\left(\cos \theta_{i}\right)\right\rangle}{\sum_{i}\left\langle\delta\left(z_{i}-z\right)\right\rangle}
$$

involving $\cos \theta_{\perp}$. The latter quantity corresponds to the cosine between the normal to the imidazolium ring and the outward $z$ axis (see Figure 1b). The plot for $P_{2}^{\cos \theta \perp}(z)$ presents a sharp drop at $z \approx 20 \AA$, a fact that would confirm that the angles between the planes of the imidazolium rings and that of the interface are close to $\sim 70^{\circ}$.

To complete our description, it will be useful to also consider the snapshot shown in Figure 1 of the Supporting Information, SI, section, which corresponds to a top view of the interface. One can clearly observe that the local density of hydrophobic groups at the interface is not enough to provide full coverage and, consequently, the exposed groups include anions and, to a lesser extent, imidazolium rings as well. This concludes our preliminary analysis of the bare interfaces and we will now move to the examination of the adsorption of the nonionic surfactant $\mathrm{C}_{12} \mathrm{E}_{5}$.

In Figure 5, we present results for $\rho_{\alpha}(z)$, at low (top panel) and high (middle panel) surface coverages. For the detergent, the plots correspond to averages taken over sites lying at the two main segments of the molecule: (i) the hydrophobic tails, comprising sites from $C_{1}$ up to $C_{12}$ and (ii) the complementary hydrophilic sections, that includes contributions from sites lying intermediate between the central $\mathrm{O}_{13}$ up to the distal $\mathrm{O}_{28}$ site (see Figure 1c for site labeling). The plots in the top panel correspond to a relatively low surface coverage, $\rho_{\text {surf }}=7.1 \times$ $10^{-3} \AA^{-2}$, which roughly corresponds to an average distance between headgroups, $l_{\mathrm{hh}} \approx \rho_{\text {surf }}^{-1 / 2} \approx 12 \AA$, still comparable to the typical length associated to the tail-to-head distance of a surfactant molecule. Under these circumstances, the density profiles for substrate species do not exhibit relevant modifications, when compared to those for bare interfaces. However, the profiles for the hydrophilic head and hydrophobic tail sections of the surfactants look both Gaussian-like of the type:

$$
\rho_{\alpha} \propto \exp \left[-\frac{4\left(z-\bar{z}_{\alpha}\right)^{2}}{\sigma_{\alpha}^{2}}\right]
$$

moreover, one also observes that the hydrophilic segment is immersed "deeper" into the underlying substrate, whereas the hydrophobic tails would remain entangled with the butyl chains of the $[\mathrm{BMIM}]^{+}$groups.

Results for a coverage approximately three times larger than the previous one, $\rho_{\text {surf }}=0.02 \AA^{-2}$, are presented in the middle panel of Figure 5. In this regime, three important modifications are clearly perceptible: (i) a complete loss of the original surface structure of the substrate; (ii) a considerable widening of the overall interface (note that the loss of uniformity in the densities of the different IL components stretches from $z \approx 12$ $\AA$ out to $z \approx 40 \AA$, away from the center of the slab); and (iii) a shift of the average positions, $\bar{z}_{\alpha}$ of the two surfactant moieties toward longer distances.

We can gauge the previous observations on more quantitative grounds by evaluating additional geometrical parameters related to the former density distributions. The results are presented in Table 1 of the SI section, in which we have included information about three sets of relevant parameters: (i) the above-mentioned widths of the Gaussian distributions associated with the detergent segments obtained from fits of the simulation results; (ii) the RTIL interface widths $\Delta z$, computed from the region along which the local density departs from the bulk value in the usual $90-10 \%$ interval and (iii) the average positions of the hydrophobic and hydrophilic segments in the surfactant molecules relative to the localization of the interface $\delta z_{\alpha}=\bar{z}_{\alpha}-z_{1 / 2}$. In the latter equality, $z_{1 / 2}$ corresponds to the distance at which the overall ionic liquid density falls down to half its bulk value, i.e., $\rho_{\alpha}\left(z_{1 / 2}\right) / \rho_{\text {bulk }}=1 / 2$.

Before getting into the analysis of these sets of data, it will be useful to bring into consideration an additional parameter related with the geometry of the intramolecular arrangement of the surfactants. We are referring to tilt angle of the type:

$$
\cos \theta_{\alpha}=\frac{\mathbf{r}_{\alpha} \cdot \hat{\mathbf{z}}}{\left|\mathbf{r}_{\alpha}\right|}
$$

(where $\alpha=\mathrm{h}, \mathrm{t}$ ) identifies head and tail segments in the surfactants and $\hat{\mathbf{z}}$ represents a unit vector along the $z$-direction (see schemes in Figure 6a). Results for the distributions for cos $\theta_{\alpha}$ are depicted in the bottom panels of Figure 6.

Armed with this comprehensive piece of information, one can conclude on the following remarks: (i) the increment of $\rho_{\text {surf }}$ promotes a sensible widening of the interface from $\Delta z \approx$ $10 \AA$ at low surface densities up to $\Delta z \approx 20 \AA$ for $\rho_{\text {surf }} \approx 0.02$ $\AA^{-2}$; (ii) the average positions of the head and tail groups with respect to the location of the interface, $\delta z_{\alpha}$, are shifted toward more external, i.e., more exposed, positions, with practically no modifications in the corresponding distribution widths $\sigma_{\alpha}$. This trend is self-evident for the headgroup case as $\delta z_{\mathrm{h}}$ shifts from $-5.3 \AA$ for system S1, up to $+1.7 \AA$ for system S4; (iii) the behavior of the orientational correlations of head and tail groups at the surface differs at a qualitative level: note that the changes in the orientations of the former segments can be barely perceived, whereas the tilts of the tail groups show a steady tendency toward a more perpendicular alignment, as the surface density increases.

A similar reduction in the extent of surfactant penetration has been observed by Shi et al. ${ }^{46}$ in their molecular dynamics 

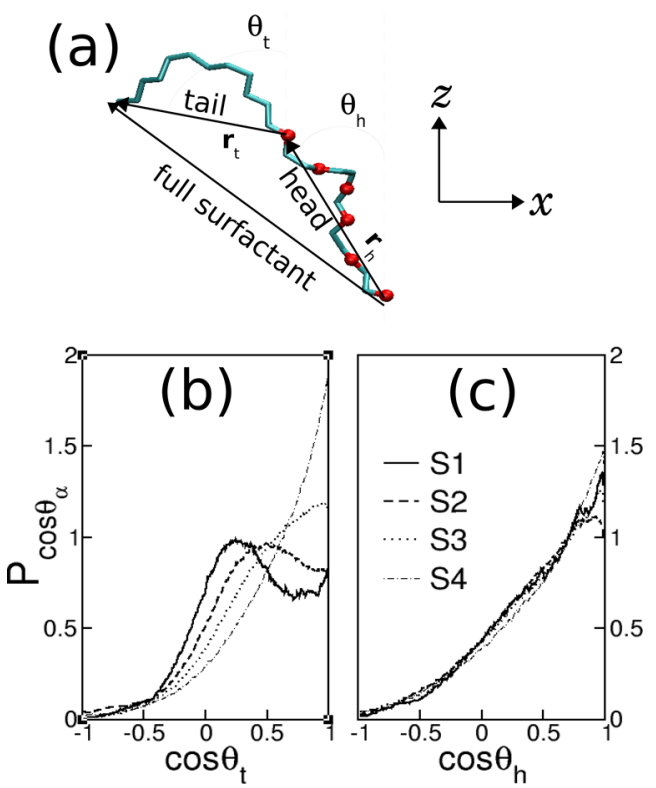

Figure 6. Top panel: Schematic representation of a $\mathrm{C}_{12} \mathrm{E}_{5}$ surfactant. $\theta_{\mathrm{h}}$ and $\theta_{\mathrm{t}}$ are the head and tail angles, respectively, with respect to $z$, the direction perpendicular to the interface. Bottom panels: probability distribution for the surfactant's tail (b) and headgroup (c) tilt angles, for the vacuum/RTIL simulated systems.

simulations on water/vacuum interfaces with increasing amounts of $\mathrm{C}_{12} \mathrm{E}_{6}$ at the interface. At the highest surface density considered $\left(0.02 \AA^{-2}\right)$ not only the tail groups but also a sizable portion of the head groups are pulled out of the water phase, exhibiting positive values of $\delta z_{\mathrm{h}}$. Moreover, the preference of head and tail segments of $\mathrm{C}_{12} \mathrm{E}_{5}$ for more external locations at high surfactant densities looks more pronounced at water/vacuum than at RTIL/vacuum interfaces. These effects are manifested by contrasting $\delta z_{\mathrm{h}}=+1.3 \AA$ (system W3) vs $\delta z_{\mathrm{h}}=-3.2 \AA$ (system S3) and $\delta z_{\mathrm{t}}=6.8 \AA$ (system W3) vs $\delta z_{\mathrm{t}}=3.7 \AA$ (system S3), which, in turn, could be ascribed to the more compact surface structure due to the hydrogen bond network at the water/vacuum interface.

So far, our simulation results have shown that the inhomogeneities in the different density fields do not span exclusively along the $z$-axis, but also along directions parallel to the interface plane. Note that, even in the absence of any surfactant, the top view of the interface (shown in the SI section) reveals a "patch-like" structure, in which the exposed groups include the two ionic liquid components whose outermost shells are localized at different values of $z$. This patch-like, irregular arrangement of the IL anions and cations along the interface plane, corresponds to the bare IL/vacuum interface and represents the equilibrium structure obtained from a $40 \mathrm{~ns}$ trajectory. We have checked that during the last 20 ns of the trajectory, the fluctuations around the ensemble averages did not surpass $1 \%$. The adsorption of the surfactants is likely to bring more complexity in the resulting roughness of the interface. Given these circumstances, we believe that the lack of any relevant structure in the density profiles shown in the lower panel of Figure 5 is likely to be the result of interference effects, arising from the inhomogeneities prevailing along directions not only perpendicular, but also parallel to the plane of the interface. To confirm such hypothesis, we performed a tessellation procedure ${ }^{47}$ which involved the following steps: (i) the centers of mass of the surfactant headgroups were brought to an arbitrary $z^{\prime}=0$ plane, by appropriate translations along the $z$-axis; (ii) after performing the alignment, the headgroups were considered as centers of Voronoi polygons which, in turn, define "columnar" sections oriented along the $z$-axis; and (iii) these "tubular" domains allowed an appropriate tagging of the different components, which were associated to one particular Voronoi section. In Figure 7, we present profiles for the $z^{\prime}$-dependence of the ionic liquid components, namely:

$$
\rho_{\alpha}\left(z^{\prime}\right)=\frac{1}{A}\left\langle\sum_{i=1} \delta\left(Z_{i}^{\alpha}-Z_{\mathrm{h}}-z^{\prime}\right)\right\rangle
$$

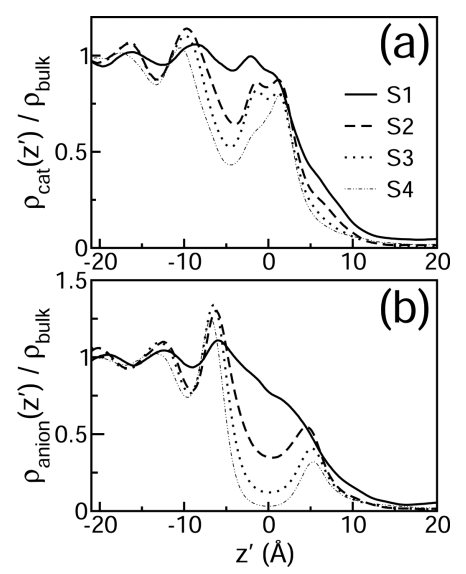

Figure 7. Normalized density profiles of (a) ionic liquid cation ring species and (b) ionic liquid anion species, with respect to the surfactant head surface, for different coverages.

The previous equation is similar to eq 1 except that, now, the sum over the index $i$ is restricted to the ionic sites of type $\alpha$ lying within the "tubular" domain defined by the headgroup with $z$-coordinate $Z_{\mathrm{h}}$. The new profiles, contrast sharply to those shown in Figure 5. Note that, except for the lowest surfactant density S1 case, the rest of the three profiles present similar qualitative characteristics. In particular, both cation and anion profiles exhibit a large extent of structure in the, $z^{\prime}<0$, inner part of the interface, with alternance of positive and negative layers. The $z^{\prime} \approx 0 \AA$ region is practically devoid of anionic species and represents a layer in which surfactant headgroups and $[\mathrm{BMIM}]^{+}$species prevail. Finally at the outermost, $z^{\prime} \approx 5-7 \AA$, shell, one still observes a remnant $\left[\mathrm{PF}_{6}\right]^{-}$layer, even at high surfactant concentrations.

Before closing this section, it will be instructive to make a brief comparative analysis between the previous results and an additional set related to the adsorption at a relatively high surface concentration, $\rho_{\text {surf }}^{-1}=78 \AA^{2}$, of two typical ionic surfactants, SDS and DTAC, at the RTIL/interface (systems $S 5^{*}$ and $\mathrm{S}^{*}$, respectively). To facilitate the direct comparison, in Figure 8, we present the density profiles for the different scenarios. The variety of relevant length scales controlling the resulting structures precludes a direct analysis at a first glance. Still, a closer look reveals a few relevant features: (i) first, in the three different RTIL slabs, one can observe that the outer, most exposed sites of the slab-plus-surfactant structures are those comprising the hydrophobic tails of both, the detergents (hydrophobic tails) and the RTIL cations (see profiles for C13 in $[\mathrm{BMIM}]^{+}$); (ii) in addition, the adsorption of the nonionic surfactant leads to a sensible loss of structure along the $z-$ 


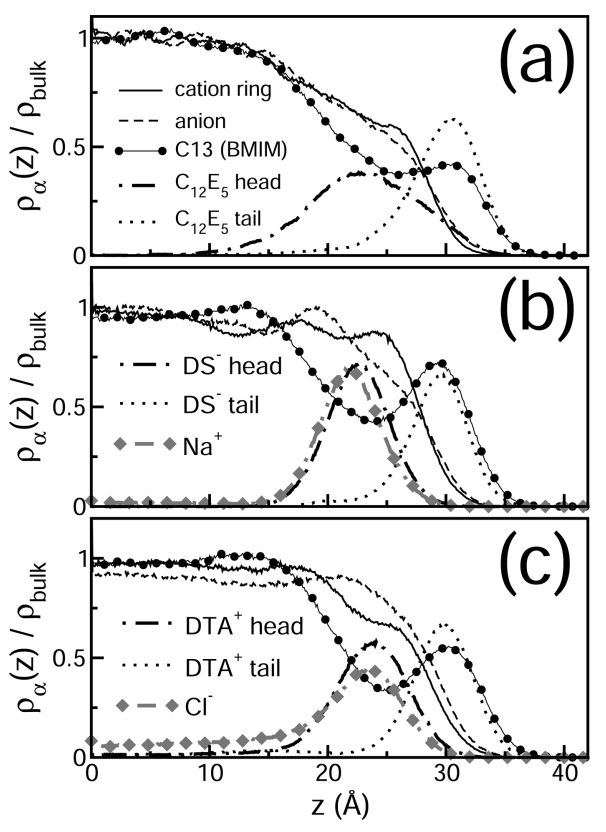

Figure 8. Normalized density profiles of different species along $z$, at a surface coverage of $\rho_{\text {surf }}^{-1}=78 \AA^{2}$, for the systems containing: (a) nonionic $\mathrm{C}_{12} \mathrm{E}_{5}$, (b) anionic SDS, and (c) cationic DTAC surfactants.

direction. Note that, with the exception of the abovementioned external shell, the rest of the density profiles in panel (a) look practically structureless, when compared to their counterparts depicted, for example, in panels (b) and (c); and (iii) a subtle inversion in the surface ordering along the $z$ direction between the cation rings (solid lines) and the $\left[\mathrm{PF}_{6}\right]^{-}$ (dashed lines) groups can also be perceived in panels (b) and (c), most likely dictated by the sign changes of the net charge of the corresponding headgroups; (iv) the ionic liquid seems to act as a "better solvent" for the $\mathrm{Cl}^{-}$anions, compared to the $\mathrm{Na}^{+}$case. Note that in the latter case (panel (b)), practically all the counterions remain in the close vicinity of the polar surfactant heagroups, while in panel (c), one observes a sizable fraction of free $\mathrm{Cl}^{-}$species in inner regions of the slab. The entries of the lower rows of Table 1 in the SI section provide additional elements that corroborate some of the previous assertions: for example, a sensible reduction of the interface width $\Delta z$ from $\sim 17 \AA$ (system S3) down to $\sim 10-11 \AA$ (systems $\mathrm{S5}^{*}$ and $\mathrm{S6}^{*}$ ) and a similar contraction in the dispersion of the headgroup locations, i.e., $\sigma_{\mathrm{h}} \approx 11 \AA$ (system S3) and $\sigma_{\mathrm{h}} \approx 6 \AA$ (systems S5* and S6*).

At this point, we would like to bring into consideration an interesting observation that derives from recent surface tension and X-ray photoelectron spectroscopy experiments performed by Chen et al. $^{48}$ on RTIL/air interfaces hosting ionic surfactants. The authors report an ionic exchange between surfactant counterions and RTIL ions of similar charge. As such, the results from these experiments would suggest significant concentration fluctuations along the mesoscopic, $\sim 30$ A depth, external strip, which would be practically devoid of counterion species. This deficit, in turn, would be compensated by an enhancement of the local concentration of the RTIL ion of similar charge.

Clearly, the results depicted in Figure 8 show not signs of an equivalent feature. Our results show that a major fraction of surfactant counterions remains bounded to the surfactant heads. One can invoke several arguments to explain this discrepancy: first, the obvious differences in the RTILs and surfactants examined. At this point, one could speculate about the possibility that the ionic exchange effect observed experimentally would not be a universal behavior for any RTIL/surfactant combination, but a particular feature derived from the specific choice of the species employed in the experimental setup. It is important to point out the structural similarity between the surfactant and one of the RTIL moieties employed by Chen et al., ${ }^{48}$ i.e., alkyl sulfate vs $\mathrm{EtSO}_{4}^{-}$and alkyl trimethylammonium vs $\mathrm{BHEDMA}^{+}$. In this context, the observed ionic exchange could be rationalized in terms of equally balanced interactions between the counterion and the two available species of opposite charge, i.e., RTIL-moiety or surfactant. Moreover, note that the greatest exchange effects are experimentally observed for short surfactant alkyl chains, a fact that supports the hypothesis that the surfactant/RTIL-moiety structural similarity would be a key factor determining the strength of the exchange effect. Contrasting, in our $\mathrm{MD}$ experiments, these structural similarities are absent. Consequently, if our line of reasoning is valid, we should not expect to find a comparable exchange effect. In second place, finite size effects, derived from our computational capabilities, preclude the identification of such long wavelength concentration fluctuations. The verification of this phenomenon via computer simulation remains an open question that surely deserves further analysis.

In the experimental work of Misono et al., ${ }^{32}$ similar systems to those studied here were investigated by means of surface tension measurements. However, it is well documented that the computation of the surface tensions of ILs via the pressure tensor can lead to unphysical liquid-vapor surface tensions, due to the slow convergence of the equilibrium averages and finite-size effects. ${ }^{49,50}$ We did attempt to perform such calculations, but we failed to get adequate convergence. Thus, those calculations were not included in this work. In a more general context, the uncertainties associated to the predictions of this magnitude are usually non-negligible for systems composed by charged entities. For example, even in simple (a)

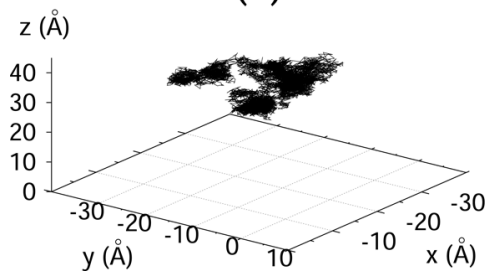

(b)

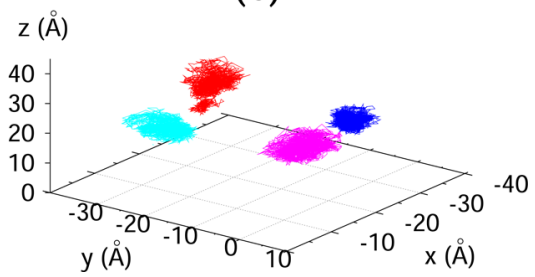

Figure 9. (a) Trajectory of the center of mass of a single nonionic surfactant $\mathrm{C}_{12} \mathrm{E}_{5}$ in the system $\mathrm{S} 3$ and (b) Trajectories of four different anionic headgroups of $\mathrm{DS}^{-}$in the S5* system (different colors correspond to different surfactant molecules). The plotted trajectories spanned over $40 \mathrm{~ns}$. 
cases such as aqueous solutions of different sodium halides, relative errors associated to the surface tensions after long simulation runs (of the order of $0.1 \mu \mathrm{s}$ ) remain in the order of $20 \%{ }^{51}$ Such limitation precluded us to obtain physically meaningful values for the surface tension, results that could be directly contrasted to experimental measurements.

3.2. Dynamics. Our dynamical analysis will focus on the mobility of the substrate and the surfactants at the interface. To easily gain an idea about the characteristics of such dynamics, in Figure 9, we present two sketches corresponding to trajectories of the centers of mass of one randomly chosen $\mathrm{C}_{12} \mathrm{E}_{5}$ molecule in system S3, (panel (a)) and four nearby anionic headgroups in system S5* (panel (b)), taken along a fairly long, $40 \mathrm{~ns}$, time span. Note that, in the former case, the coupling with the substrate is sufficiently weak so that the nonionic surfactant exhibits displacements across distances comparable to onequarter of the simulation box, say, $\sim 15 \AA$. This behavior should be contrasted with the ones for the trajectories of the $\mathrm{DS}^{-}$ headgroups, which look like those corresponding to fast oscillatory-like motions within different surface traps.

The previous observations can be brought into more quantitative grounds, by computing surface diffusion coefficients $D_{\alpha}^{\|}$, from the mean square displacements (MSD), namely:

$$
D_{\alpha}^{\|}=\lim _{t \rightarrow \infty} \frac{1}{4 t} \mathcal{R}_{\alpha}^{2}(t)
$$

where

$$
\mathcal{R}_{\alpha}^{2}=\left\langle\left[X_{i}^{\alpha}(t)-X_{i}^{\alpha}(0)\right]^{2}+\left[Y_{i}^{\alpha}(t)-Y_{i}^{\alpha}(0)\right]^{2}\right\rangle
$$

In the previous equation, the tagged $i$-th molecules were chosen from those lying at the $Z_{i}^{\alpha}>10 \AA$, interface region, at times 0 and $t$. In the SI, we have included MSD plots for the S3, $\mathrm{S5}^{*}$, and S6* systems, obtained from $200 \mathrm{~ns} \mathrm{MD}$ trajectories and spanned over 15 and $50 \mathrm{~ns}$. The log-log scale of the MSD plots shows that the diffusive linear regime is attained within the former time scale. Our estimates for the different $D_{\alpha}^{\|}$are listed in Table 2 where, as a reference, we have also included molecular dynamics results for the bulk RTIL.

Several observations about the previous set of data are worth commenting: (i) as expected, and in accordance with previous simulation analysis of different vacuum/liquid interfaces, ${ }^{56}$ the diffusion coefficients for the two IL components at the bare interface present increments, which are more marked in the

Table 2. Self-Diffusion Coefficients (in $10^{-10} \mathrm{~m}^{2} / \mathrm{s}$ )

\begin{tabular}{|c|c|c|c|}
\hline system & $D_{\mathrm{BMIM}}^{\|}$ & $D_{\mathrm{PF}_{6}}^{\|}$ & $D_{\text {surf }}^{\|}$ \\
\hline So & 0.25 & 0.23 & \\
\hline S1 & 0.22 & 0.17 & 0.20 \\
\hline $\mathrm{S} 2$ & 0.20 & 0.16 & 0.21 \\
\hline S3 & 0.19 & 0.15 & 0.19 \\
\hline S4 & 0.17 & 0.13 & 0.16 \\
\hline S5* & 0.060 & 0.035 & 0.020 \\
\hline S6* & 0.047 & 0.032 & 0.018 \\
\hline W3 & & & $\begin{array}{c}4.4 \\
(4 \pm 1)^{a}, 4.17^{b}\end{array}$ \\
\hline bulk & $\begin{array}{c}0.23 \\
\left(0.247^{c}, 0.259^{d}\right)\end{array}$ & $\begin{array}{c}0.15 \\
\left(0.190^{c}, 0.206^{d}\right)\end{array}$ & \\
\hline
\end{tabular}
Obtained for the Different Species at $T=323 \mathrm{~K}$ case of $\left[\mathrm{PF}_{6}\right]^{-}$moiety (of the order of $\sim 50 \%$, compared to the bulk value) and (ii) the addition of increasing amounts of surfactant molecules promotes a gradual reduction in the diffusive motions. Interestingly, retardation effects seem to be also more marked for the anionic species: note that, in passing from the S0 to the S4 system, the diffusion coefficient for the $\left[\mathrm{PF}_{6}\right]^{-}$species presents a $\sim 42 \%$ reduction, a drop somewhat larger than the one observed for the $[\mathrm{BMIM}]^{+}$groups, which is of the order of a $\sim 35 \%$. Still, the overall effects in the substrate derived from the presence of the $\mathrm{C}_{12} \mathrm{E}_{5}$ surfactants are, in all cases, much milder than those registered in interfaces hosting ionic surfactants where the resulting diffusion coefficients are practically four times smaller than those observed for the nonionic surfactant case (see entries for S5* and S6* systems). Concerning now the dynamics of the surfactant groups, the dynamical modifications operated at different coverages are much less marked than in the substrate, and in all cases, $D_{\text {surf }}^{\|}$ remains of the order to $\sim 0.15-0.20 \times 10^{-10} \mathrm{~m}^{2} / \mathrm{s}$. In this context, it is instructive to compare these values with the one observed if one changes the underlying substrate and/or the surfactant species: note that the diffusion coefficient $D_{\text {surf }}^{\|}$for the system S4 is practically 2 orders of magnitude smaller than that reported for $\mathrm{C}_{12} \mathrm{E}_{5}$ adsorbed at water/vacuum interfaces (system W3) and 1 order of magnitude larger than the diffusion coefficients for $\mathrm{DS}^{-}$or $\mathrm{DTA}^{+}$groups at RTIL/vacuum interfaces (systems $\mathrm{S}^{*}$ and $\mathrm{S}^{*}$, respectively).

\section{CONCLUSIONS}

The analysis presented in this work provides new insights on structural and dynamical characteristics of the adsorption of different surfactants at RTIL/vacuum interfaces. More specifically, our description focused on the surface activity of the nonionic detergent $\mathrm{C}_{12} \mathrm{E}_{5}$, whose behavior was then taken as a reference in a subsequent comparative analysis against systems involving two commonly used, ionic surfactants: SDS and DTAC.

The overall picture that emerges from our simulations reveals an intricate interface structure, which is controlled not only by the surfactant groups, but also by the surface activity of the different IL components. These complexities are manifested, most notably, in the following features: (i) first, a considerable broadening of the interface along directions perpendicular to the interface, when compared to the classical example of the bare water/vacuum one; (ii) second, a layering structure along the same direction, comprising an outermost shell-in which hydrocarbon tails of the $[\mathrm{BMIM}]^{+}$moieties prevail-adjacent to an inner surface domain, in which the populations of positively and negatively charged groups of the IL practically compensate each other; (iii) finally, a "patch"-like, irregular arrangement along directions parallel to the interface plane, revealing that the three main moieties, i.e., $\left[\mathrm{PF}_{6}\right]^{-},[\mathrm{BMIM}]^{+}$ hydrophobic tails and $[\mathrm{BMIM}]^{+}$imidazol-headgroups, remain exposed to the vacuum.

The incorporation of $\mathrm{C}_{12} \mathrm{E}_{5}$ at low surface coverages does not promote meaningful modifications in the substrate. At higher surface coverages, when the average distance between the adsorbed surfactant species becomes smaller than the tail-tohead distances, all structure in the underlying substrate practically disappears. In addition, the surfactant molecules move toward more external positions, partially coating the underlying IL phase. An analysis based on a tessellation protocol allows one to recover, in part, information about the local structure of the interface at high surface coverages: with 
respect to the positions of the surfactant headgroups, one observes alternance of positive and negative layers in inner domains and a few remnant $\left[\mathrm{PF}_{6}\right]^{-}$groups in outer ones.

Concerning similarities and differences between the abovementioned behaviors and those pertaining to the adsorption of ionic surfactants, we have verified that, in the latter cases, the original width and structure of the interface that would prevail in the absence of the surfactant, are better preserved. In all cases, at high surface coverages, the hydrophobic tails of the surfactants and the $[\mathrm{BMIM}]^{+}$groups exhibit a non-negligible degree of entanglement. In addition, the underlying IL seems to behave as a "better" solvent for the $\mathrm{Cl}^{-}$counterions, compared to the $\mathrm{Na}^{+}$species which, in turn, are found to remain bound to the surfactant headgroups.

We finally conclude with a brief comment on the dynamics at the surface. Our results suggest that the presence of increasing amounts of neutral surfactants leads to a gradual reduction in the mobility of the two IL components. This phenomenon is more marked in the case of the anionic component. Still, these overall effects look milder when compared to those derived from the adsorption of ionic detergents. The magnitude of the latter modifications are also perceived in the dynamical characteristics of the surfactant headgroups across the interface, where the changes in the corresponding diffusion coefficients in passing from the nonionic to the ionic-detergent case involve one order of magnitude reduction.

\section{ASSOCIATED CONTENT}

\section{S Supporting Information}

A top view of the interface, for the bare vacuum/RTIL system, a table containing the structural parameters computed from the density profiles and plots of the mean square displacements. This material is available free of charge via the Internet at http://pubs.acs.org

\section{AUTHOR INFORMATION}

\section{Corresponding Author}

*E-mail: DoloresElola@gmail.com.

\section{Notes}

The authors declare no competing financial interest.

\section{ACKNOWLEDGMENTS}

We are grateful to Professor Daniel Laria for several stimulating and helpful discussions, and for valuable suggestions when reading this manuscript. M.D.E. and J.R. are staff members of CONICET, Argentina.

\section{REFERENCES}

(1) Welton, T. Room temperature ionic liquids: Solvents for synthesis and catalysis. Chem. Rev. 1999, 99, 2071-2083.

(2) Angell, A. C.; Byrne, N.; Belieres, J. P. Parallel developments in aprotic and protic ionic liquids: Physical chemistry and applications. Acc. Chem. Res. 2007, 40, 1228.

(3) Greaves, T. L.; Drummond, C. J. Protic ionic liquids: Properties and applications. Chem. Rev. 2008, 108, 206.

(4) Massey, J. A.; Winnik, M. A.; Manners, I.; Chan, V. Z. H.; Ostermann, J. M.; Enchelmaier, R.; Spatz, J. P.; Möller, M. Fabrication of oriented nanoscopic ceramic lines from cylindrical micelles of an organometallic polyferrocene block copolymer. J. Am. Chem. Soc. 2001, 123, 3147-3148.

(5) Sohn, B.-H.; Choi, J.-M.; Yoo, S. I.; Yun, S.-H.; Zin, W.-C.; Jung, J. C.; Kanehara, M.; Hirata, T.; Teranishi, T. Directed self-assembly of two kinds of nanoparticles utilizing monolayer films diblock copolymer micelles. J. Am. Chem. Soc. 2003, 125, 6368-6369.
(6) Jaramillo, T. F.; Baeck, S.-H.; Roldan Cuenya, B.; McFarland, E. W. Catalytic activity of supported Au nanoparticles deposited from block copolymer micelles. J. Am. Chem. Soc. 2003, 125, 7148-7149.

(7) Khara, D. C.; Samanta, A. Rotational dynamics of positively and negatively charged solutes in ionic liquid and viscous molecular solvent studied by time-resolved fluorescence anisotropy measurements. Phys. Chem. Chem. Phys. 2010, 12, 7671.

(8) Khara, D. C.; Samanta, A. Fluorescence response of coumarin153 in $N$-alkyl- $n$-methylmorpholinium ionic liquids: Are these liquids more structured than the imidazolium ionic liquids? J. Phys. Chem. B 2012, 116, 13430.

(9) Wasserscheid, P.; Keim, W. Ionic liquids: New solutions for transition metal catalysis. Angew. Chem., Int. Ed. 2000, 39, 3772-3789.

(10) Yao, C.; Pitner, W. R.; Anderson, J. L. Ionic liquids containing the Tris(pentafluoroethyl)trifluorophosphate anion: A new class of highly selective and ultra hydrophobic solvents for the extraction of polycyclic aromatic hydrocarbons using single drop microexcitation. Anal. Chem. 2009, 81, 5054.

(11) Bates, E. D.; Mayton, R. D.; Ntai, I.; Davis, J. H., Jr. $\mathrm{CO}_{2}$ capture by task-specific ionic liquids. J. Am. Chem. Soc. 2002, 124, 926-927.

(12) Huddleston, J. G.; Rogers, R. D. Room temperature ionic liquids as novel media for clean liquid-liquid extraction. Chem. Commun. 1998, $34,1765-1766$

(13) Riisager, A.; Jorgensen, B.; Wasserscheid, P.; Fehrmann, R. First application of supported ionic liquid phase (SILP) catalysis for continuous methanol carbonylation. Chem. Commun. 2006, 42, 994996.

(14) Riisager, A.; Fehrmann, R.; Flicker, S.; van Hal, R.; Haumann, M.; Wasserscheid, P. Very stable and highly regioselective supported ionic-liquid-phase (SILP) catalysis: Continuous-flow fixed-bed hydroformylation of propene. Angew. Chem., Int. Ed. 2005, 44, 815.

(15) Endres, F.; El Abedin, S. Z. Air and water stable ionic liquids in physical chemistry. Phys. Chem. Chem. Phys. 2006, 8, 2101-2116.

(16) Lynden-Bell, R. M. Gas/liquid interfaces of room temperature ionic liquids. Mol. Phys. 2003, 101, 2625.

(17) Wang, Y.; Voth, G. A. Unique spatial heterogeneity in ionic liquids. J. Am. Chem. Soc. 2005, 127, 12192-12193.

(18) Bhargava, B. L.; Balasubramanian, S. Layering at an ionic liquidvapor interface: A MD simulation study of [bmim][PF6]. J. Am. Chem. Soc. 2006, 128, 10073-10078.

(19) Lynden-Bell, R. M.; Del Pópolo, M. G. Simulation of the surface structure of butylmethylimidazolium ionic liquids. Phys. Chem. Chem. Phys. 2006, 8, 949.

(20) Sloutskin, E.; Lynden-Bell, R. M.; Balasubramanian, S.; Deutsch, $\mathrm{M}$. The surface structure of ionic liquids: Comparing simulations with X-ray measurements. J. Chem. Phys. 2006, 125, 174715.

(21) Wang, Y.; Jiang, W.; Yan, T.; Voth, G. A. Understanding ionic liquids through atomistic and coarse-grained molecular dynamics simulations. Acc. Chem. Res. 2007, 40, 1193-1199.

(22) Lynden-Bell, R. M.; del Pópolo, M. G.; Youngs, T. G. A.; Kohanoff, J.; Hanke, C. G.; Harper, J. B.; Pinilla, C. C. Simultions of ionic liquids, solutions and interfaces. Acc. Chem. Res. 2007, 40, 11381145.

(23) Gannon, T. J.; Law, G.; Watson, P. R.; Carmichael, A. J.; Seddon, K. R. First observation of molecular composition and orientation at the surface of a room temperature ionic liquid. Langmuir 1999, 15, 8429.

(24) Law, G.; Watson, P. R. Surface orientation in ionic liquid. Chem. Phys. Lett. 2001, 345, 1-4.

(25) Baldelli, S. Influence of water on the orientation of cations at the surface of a room temperature ionic liquid: A sum frequency generation vibrational spectroscopic study. J. Phys. Chem. B 2003, 107, 6148-6152.

(26) Sloutskin, E.; Ocko, B. M.; Tamam, L.; Kuzmenko, I.; Gog, T.; Deutsch, M. Surface layering in ionic liquids: An X-ray reflectivity study. J. Am. Chem. Soc. 2005, 127, 7796-7804.

(27) Santos, C. S.; Baldelli, S. Surface orientation of 1-methyl, 1-ehyl, and 1-butyl-3-methylimidazolium methyl sulfate as probed by sum 
frequency generation vibrational spectroscopy. J. Phys. Chem. B 2007, $111,4715-4723$.

(28) Waring, C.; Bagot, P. A. J.; Slattery, J. M.; Costen, M. L.; McKendrick, K. G. O(3P) atoms as a probe of surface ordering in 1alkyl-3- methylimidazolium-based ionic liquids. J. Phys. Chem. Lett. 2010, 1, 429.

(29) Niga, P.; Wakeham, D.; Nelson, A.; Warr, G. G.; Rutland, M.; Atkin, R. Structure of ethylammonium nitrate surface: An X-ray reflectivity and vibrational sum frequency spectroscopy study. Langmuir 2010, 26, 8282-8288.

(30) Wang, S.; Ma, J. Surface property of nonionic surfactant triton X-100 in an ionic liquid. J. Disper. Sci. Technol. 2009, 30, 1395-1398.

(31) Wakeham, D.; Niga, P.; Warr, G. G.; Rutland, M. W.; Atkin, R. Nonionic surfactant adsorption at the ethylammonium nitrate surface: A neutron reflectivity and vibrational sum frequency spectroscopy study. Langmuir 2010, 26, 8313-8318.

(32) Misono, T.; Sakai, H.; Sakai, K.; Abe, M.; Inoue, T. Surface adsorption and aggregate formation of nonionic surfactants in a room temprature ionic liquids. J. Colloid Interface Sci. 2011, 358, 527.

(33) Evans, D. F.; Yamauchi, A.; Wei, G. J.; Bloomfield, V. A. Micelle size in ethylammonium nitrate as determined by classical and quasielastic light scattering. J. Phys. Chem. 1983, 87, 3537.

(34) Inoue, T.; Yamakawa, H. Micelle formation of nonionic surfactants in room temperature ionic liquids. J. Colloid Interface Sci. 2011, 356, 798-802.

(35) Gao, Y.; Li, N.; Li, X.; Zhang, S.; Zheng, L.; Bai, X.; Yu, L. Microstructures of micellar aggregations formed within 1-butyl-3methylimidazolium type ionic liquids. J. Phys. Chem. B 2009, 113, 123-130.

(36) Morrow, T. I.; Maginn, E. J. Molecular dynamics study of the ionic liquid 1-nbutyl-3methylimidazolium hexafluorophosphate. J. Phys. Chem. B 2002, 106, 12807.

(37) Canongia Lopes, J. N.; Deschamps, J.; Pádua, A. A. H. Modeling ionic liquids using a systematic all-atom force field. J. Phys. Chem. B 2004, 108, 2038.

(38) Liu, Z.; Huang, S.; Wang, W. A refined force field for molecular simulation of imidazolium-based ionic liquids. J. Phys. Chem. B 2004, $108,12978$.

(39) Zhong, X.; Liu, Z.; Cao, D. Improved classical united-atom force field for imidazolium-based ionic liquids: tetrafluoroborate, hexafluorophosphate, mehylsulfate, trifluoromethylsulfonate, acetate, trifluoroacetate, and bis(trifluoromethylsulfonyl)amide. J. Phys. Chem. B 2011, $115,10027$.

(40) MacKerell, J. A. D.; Banavali, N.; Foloppe, N. Development and current status of the CHARMM force field for nucleic acids. Biopolymers 2001, 56, 257.

(41) Lei, S.; Zhang, J.; Huang, J.-B. Promotion of the surface activity and aggregation ability of SDS in aqueous solution by ionic liquid [bmim] $\left[\mathrm{BF}_{4}\right]$. Acta Phys.-Chim. Sin. 2007, 23, 1657.

(42) Phillips, J. C.; Braun, R.; Wang, W.; Gumbart, J.; Tajkhorshid, E.; Villa, E.; Chipot, C.; Skeel, R. D.; Kale, L.; Schulten, K. Scalable MD with namd. J. Comput. Chem. 2005, 26, 1781.

(43) Darden, T. A.; York, D. M.; Pedersen, L. G. Particle mesh Ewald-An $N \cdot \log (N)$ method for Ewald sums in large systems. J. Chem. Phys. 1993, 98, 10089.

(44) Essmann, U.; Perera, L.; Berkowitz, M. L.; Darden, T.; Lee, H.; Pedersen, L. G. A smooth particle mesh Ewald method. J. Chem. Phys. 1995, 103, 8577.

(45) Gu, Z.; Brennecke, J. F. Volume expansivities and isothermal compressibilities of imidazolium and pyridinium-based ionic liquids. $J$. Chem. Eng. Data 2002, 47, 339.

(46) Shi, L.; Tummala, R.; Striolo, A. C12E6 and SDS surfactants simulated at the vacuum/water interface. Langmuir 2010, 26, 54625474.

(47) Pandit, A.; Bostick, D.; Berkowitz, M. L. An algorithm to describe molecular scale rugged surfaces and its application to the study of a water/lipid bilayer interface. J. Chem. Phys. 2003, 119, 2199.
(48) Chen, L. G.; Lerum, R. V.; Aranda-Espinoza, H.; Bermudez, H. Surfactant-mediated ion exchange and charge reversal at ionic liquid interfaces. J. Phys. Chem. B 2010, 114, 11502.

(49) González-Melchor, M.; Bresme, F.; Alejandre, J. Molecular dynamics simulations of the surface tension of ionic liquids. J. Chem. Phys. 2005, 122, 104710.

(50) Chang, T. M.; Dang, L. X. Computational studies of structures and dynamics of 1,3-dimethylimidazolium salt liquids and their interfaces using polarizable potential models. J. Phys. Chem. A 2009, $113,2127$.

(51) dos Santos, D. J. V. A.; Müller-Plathe, F.; Weiss, V. C. Consistency of ion adsorption and excess surface tension in molecular dynamics simulations of aqueous salt solutions. J. Phys. Chem. C 2008, 112, 19431-19442.

(52) Cuny, V.; Antoni, M.; Arbelot, M.; Liggieri, L. Structural properties and dynamics of $\mathrm{C}_{12} \mathrm{E}_{5}$ molecules adsorbed at water/air interfaces: A molecular dynamics study. Colloids Surf. A 2008, 323, 180.

(53) Eastoe, J.; Dalton, J. S.; Rogueda, P. G. A.; Crooks, E. R.; Pitt, A. R; Simister, E. A. Dynamic surface tensions of nonionic surfactant solutions. J. Colloid Interface Sci. 1997, 188, 423.

(54) Tukuda, H.; Hayamizu, K.; Ishii, K.; Bin Hasan Susan, A. Physicochemical properties and structures of room temperature ionic liquids. 1. Variation of anionic species. J. Phys. Chem. B 2004, 108, 16593.

(55) Umecky, T.; Kanakubo, M.; Ikushima, Y. Effects of alkyl chain on transport properties in 1-alkyl-3-methylimidazolium hexafluorophosphate. J. Mol. Liq. 2005, 119, 77.

(56) Liu, P.; Harder, E.; Berne, B. J. On the calculation of diffusion coefficients in confined fluids and interfaces with an application to the liquid-vapor interface of water. J. Phys. Chem. B 2004, 108, 65956602 . 